\title{
Neutrophil extracellular traps (NETs) - formation and implications*
}

\author{
Marcin Zawrotniak and Maria Rapala-Kozik \\ Department of Analytical Biochemistry, Faculty of Biochemistry, Biophysics and Biotechnology, Jagiellonian University, Kraków, Poland
}

Neutrophils are cells of the immune system which freely circulate in blood vessels and are recruited to the inflammation sites when the human organism responds to microbial infections. One of the mechanisms of neutrophil action is the formation of neutrophil extracellular traps (NETs) The process of NET generation, called netosis, is a specific type of cell death, different from necrosis and apoptosis. NETs are formed by neutrophils upon contact with various bacteria or fungi as well as with activated platelets or under the influence of numerous inflammatory stimuli, and this process is associated with dramatic changes in the morphology of the cells. The main components of NETs, DNA and granular antimicrobial proteins, determine their antimicrobial properties. The pathogens trapped in NETs are killed by oxidative and non-oxidative mechanisms. On the other hand, it was also discovered that chromatin and proteases released into the circulatory system during NET formation can regulate procoagulant and prothrombotic factors and take part in clot formation in blood vessels. NETs have also been detected in lungs where they are involved in chronic inflammation processes in ALI/ARDS patients. Moreover, DNA-proteins complexes have been found in the airway fluids of cystic fibrosis patients where they can increase the viscosity of the sputum and have a negative impact on the lung functions. The DNA-complexed granular proteins and other proteins released by neutrophils during netosis lead to autoimmunity syndromes such as systemic lupus erythematosus (SLE), small-vessel vasculitis (SVV) or autoimmune diseases associated with the formation of autoantibodies against chromatin and neutrophil components. A possible involvement of NETs in metastasis is also considered.

Key words: neutrophils, neutrophil extracellular traps, netosis, lung disease, autoimmune disease, thrombosis, cancer

Received: 25 April, 2013; accepted: 05 June, 2013; available on-line: 01 July, 2013

\section{INTRODUCTION}

Neutrophils are important components of innate immunity necessary to maintain homeostasis of the organism. They are short-lived polymorphonuclear granulocytes which constitute a primary defence against microbial infections. In acute inflammation, neutrophils circulating with the bloodstream are rapidly recruited to the site of infection, in response to chemotactic factors released by pathogens or host cells. After attachment to the endothelium, neutrophils leave blood vessels and move toward the site of infection, sensing the chemotactic gradient. At the inflammatory site, activated immune cells acquire the ability to kill pathogens. To fulfill that task, neutrophils use a number of strategies such as phagocytosis, degranulation and the recently discovered formation of extracellular traps. During phagocytosis, internalized pathogens are translocated to phagosomes where the antimicrobial factors derived from granules and reactive oxygen species (ROS) create a killing environment for pathogens. The second mechanism, degranulation, is similar to phagocytosis, but rather than being engulfed the pathogens are killed extracellularly by the same antimicrobial factors which are in part released outside the cell. The neutrophil extracellular traps (NETs) can be released by neutrophils in a process called netosis. NETs are a special kind of trap formed by decondensed chromatin fibres decorated with antimicrobial factors delivered by the granules. The main function of NETs is trapping and killing of pathogens (Brinkmann et al., 2004).

\section{NETOSIS}

Netosis is a specific type of cell death different from both necrosis and apoptosis but its mechanism is still poorly understood (Fuchs et al., 2007). The most important feature specific to NETs is the presence of neutrophil nuclear DNA fibres in the extracellular space. NETs are produced by neutrophils in contact with pathogens such as bacteria, fungi, viruses and protozoa (Table 1), with a variety of host factors such as activated platelets or inflammatory stimuli or with chemical compounds (e.g. phorbol-12-myristate-13-acetate) (Brinkmann et al., 2004).

e-mail: maria.rapala-kozik@uj.edu.pl

*Presented at 40th Jubilee Winter School of the Faculty of Biochemistry, Biophysics and Biotechnology of the Jagiellonian University "Contemporary insights into cancer. Risk, perspectives, expectations", February 16-21, 2013, Zakopane, Poland.

Abbreviations: ALI, acute lung injury; ANCA, anti-neutrophil cytoplasmic antibodies; ARDS, acute respiratory distress syndrome; CF, cystic fibrosis; CGD, chronic granulomatous disease; Dnase, deoxyribonuclease 1; DVT, deep vein thrombosis; Ets, extracellular traps; fMLP, N-formyl-methionyl-leucyl-phenylalanine; FVII/VIla, bloodcoagulation factor VII; G-CSF, granulocyte colony-stimulating factor; GM-CSF, granulocyte-macrophage colony-stimulating factor; IC, immune complexes; IL-8, Interleukin 8; LPS, lipopolysaccharide; MAC-1, macrophage-1 antigen, integrin aM 32 ; $\mathrm{MDC}$, myeloid dendritic cell; MPO, myeloperoxidase; NE, neutrophil elastase; NETs, neutrophil extracellular traps; PAD4, peptidylarginine deiminase 4; PHOX, phagocyte NADPH oxidase; PMA, phorbol-12-myristate-13acetate; PR3, proteinase 3; RBC, red blood cell; rhDNase, recombinant human DNase; ROS, reactive oxygen species; SLE, systemic lupus erythematosus; SP-A, SP-B, SP-D, surfactant proteins; SVV, small-vessel vasculitis; TF, tissue factor; TLR, toll-like receptor; TNF-a, tumor necrosis factor; TRALI, transfusion-related acute lung injury; VWF, von Willebrand factor. 
Table 1. Microbial and chemical factors which stimulate the formation of NETs (Guimarães-Costa et al., 2012).

\begin{tabular}{|c|c|}
\hline Microbial factors & Chemical factors \\
\hline Aspergillus fumigatus & $\delta$-Toxin from Staphylococcus epidermidis \\
\hline Candida albicans & Antibodies \\
\hline Cryptococcus gattii & Calcium ions \\
\hline Cryptococcus neoformans & Glucose oxidase \\
\hline Eimeria bovis & $\mathrm{GM}-\mathrm{CSF}+\mathrm{C} 5 \mathrm{a}$ \\
\hline Enterococcus faecalis & GM-CSF + LPS \\
\hline Escherichia coli & Hydrogen peroxide \\
\hline Haemophilus influenzae & Interferon- $a+C 5 a$ \\
\hline Helicobacter pylori & Interleukin 8 \\
\hline Klebsiella pneumoniae & Lipopolysaccharide (LPS) \\
\hline Lactococcus lactis & M1 protein \\
\hline Leishmania amazonensis donovani/major/chagasi & Nitric oxide \\
\hline Listeria monocytogenes & Phorbol-12-myristate-13-acetate (PMA) \\
\hline Mannheimia haemolytica & PMA + ionomycin \\
\hline Mycobacterium tuberculosis/canettii & Platelet activating factor \\
\hline Serratia marcescens & TLR-4 \\
\hline Shigella flexneri & TNF-a \\
\hline \multicolumn{2}{|l|}{ Staphylococcus aureus } \\
\hline \multicolumn{2}{|l|}{ Streptococcus dysgalactiae } \\
\hline \multicolumn{2}{|l|}{ Streptococcus pneumonia } \\
\hline Yersinia enterocolitica & \\
\hline
\end{tabular}

Several factors and events were proposed to be engaged in netosis. The timing of NET formation and the dependency on ROS production, NET composition and the involvement of cell death differ depending on the type of stimulus used in the NET studies. Nevertheless, some of the fundamental steps have been determined (Papayannopoulos \& Zychlinsky, 2009; Parker \& Winterbourn, 2012). During activation, neutrophils produce large amounts of ROS through the action of NADPH oxidase (PHOX) Fuchs et al. (2007) have presented evidence that ROS are also initiators of NET production. For example, neutrophils from chronic granulomatous disease (CGD) patients cannot produce NETs as CGD results from a mutation in PHOX subunits that affects the enzyme activity. However, treatment of CGDneutrophils with $\mathrm{H}_{2} \mathrm{O}_{2}$ restored their ability to form NETs (Fuchs et al., 2007; Nishinaka et al., 2011).

After stimulation, the neutrophil chromatin undergoes decondensation followed by mixing of euchromatin and heterochromatin (Fuchs et al., 2007). This process is mediated by enzymes stored in the azurophilic granules, neutrophil elastase (NE) and myeloperoxidase (MPO), which are relocated to the nucleus by a yet unknown mechanism. First, NE degrades the linker histone $\mathrm{H} 1$ and the core histones, leading to chromatin decondensation which is enhanced by MPO, independent of the enzymatic activity of the latter (Papayannopoulos et al., 2010; Metzler et al., 2011). Moreover, during NET formation, histone $\mathrm{H} 3$ undergoes a modification (the "citrullination") that converts arginine residues to citrulline (Wang et al., 2009; Leshner et al., 2012; Neeli \& Radic, 2013). The citrullination of histones is catalysed by peptidylarginine deiminase 4 (PAD4) which is localized in the nucleus of neutrophils. It was shown that neutrophils isolated from PAD4-knockout mice lost their ability to release NET and histone hypercitrullination was not detectable (Li et al., 2010). Subsequently, the nuclear membrane is damaged, chromatin expands inside
Figure 1. Mechanism of NET release.

Stimulation of receptors (A) by triggers (e.g. bacteria, fungi, viruses, parasites, chemical factors like PMA or LPS) leads to the adherence of neutrophils to endothelium and to chromatin decondensation due to histone cleavage by NE and MPO and histone hypercitrullination by PAD4 (B). In the final phase, NETs are released and trap bacteria $(\mathbf{C})$. 
the cell and is mixed with granular antimicrobial factors. Finally, the cell membrane breaks releasing NETs (Brinkmann \& Zychlinsky, 2012).

All these processes presented above determine a new type of neutrophil death but NETs can be also released within minutes from living neutrophil cells through an oxidant-independent mechanism as it was demonstrated in S. aureus infection (Pilsczek et al., 2010; Yipp et al., 2012). Given that other cell types (mast cells, basophils and macrophages) are also able to form extracellular traps (ETs) (Goldmann \& Medina, 2013), this new defence process, collectively named etosis, still remains a mystery.

\section{NET antimicrobial actions}

NETs are able to trap almost all types of pathogens, even those so large that they cannot be phagocytosed, including gram-positive and gram-negative bacteria, yeasts, viruses and protozoan parasites (Lu et al., 2012). The trapping within the DNA fibres prevents the spread of microorganisms over the body and facilitates a higher concentration of antimicrobial factors at the site of infection (Brinkmann et al., 2004). The trapping occurs through charge interactions between the pathogen cell surface and NET components. Their antimicrobial functions are represented by proteins originating from both the granules and the cytoplasm i.e., not only NE, histone and MPO but also cathepsin G, proteinase 3 (PR3), lactoferrin, calprotectin and antimicrobial peptides such as defensins or the cationic antimicrobial protein-derived peptide LL37 (Urban et al., 2009). NET-associated proteases (NE or PR3) can inactivate and kill pathogens by cleaving their virulence factors (Brinkmann et al., 2004). LL37 and histones can disintegrate the pathogen cell membranes, challenging the pathogen viability (Cho et al., 2009; Méndez-Samperio, 2010). The activity of MPO is essential for killing $S$. aureus (Parker \& Winterbourn, 2012), but fungal growth is restricted by neutrophil proteins which act in ion sequestration such as lactoferrin or calgranulin (Farnaud \& Evans, 2003; Urban et al., 2009).

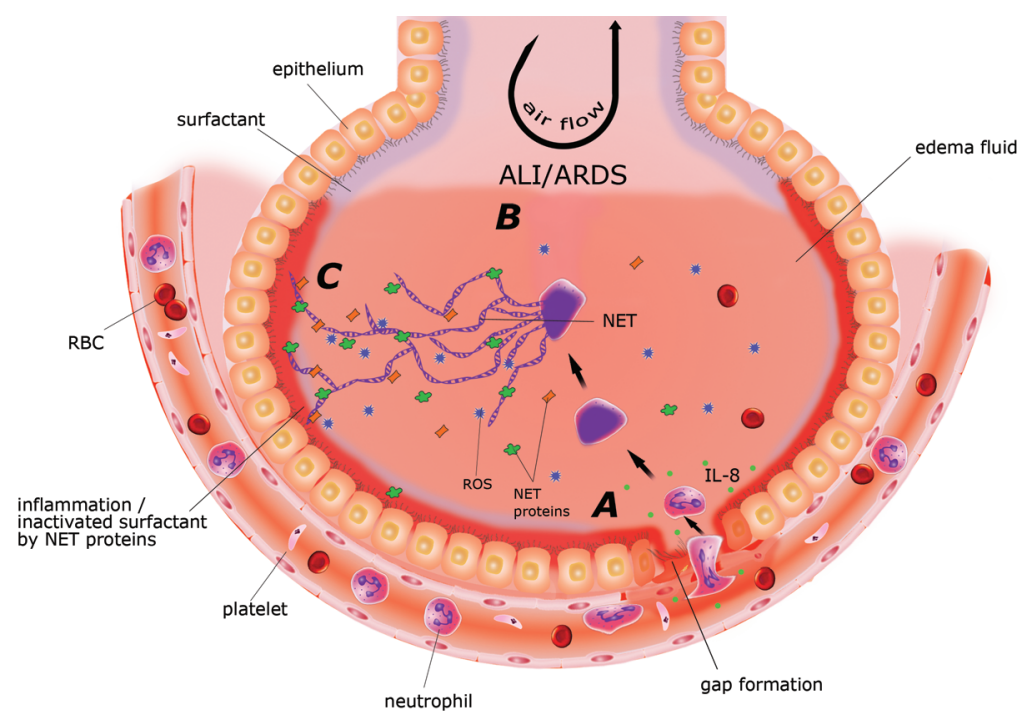

Figure 2. Alveolar space in ALI/ARDS.

Injury of alveolar epithelial cells increases the permeability of the barrier between the alveolar space and blood vessels. Additionally, the epithelium releases IL-8. Those conditions promote a leakage of neutrophils with the edema fluid into the alveolar space $(\mathbf{A})$. Inside alveoli neutrophils release NETs in response to stimulating factors (B). The NET components, such as proteinases or ROS, cause secondary epithelial cell damage, leading to chronic inflammation (C).
However, NETs are not perfect in microbe killing and some pathogens have evolved mechanisms to evade NETs. Such mechanism, identified in $S$. aureus, or $S$. pyogenes is based on the secretion of endonucleases which degrade DNA (Beiter et al., 2006). On the other hand, pathogens may also avoid trapping by changing their surface charge or making a polysaccharide capsule such as that formed by S. pneumonia (Wartha et al., 2007).

\section{NETS AND INFLAMMATION}

Besides the proposed antibacterial function of NETs, their ineffective clearance or excessive formation can cause several pathological effects. NET formation was observed during chronic inflammatory disease (atherosclerosis), autoimmune diseases (SLE), in diverse forms of vasculitis, thrombosis, transfusion-related acute lung injury (TRALI) and in cancer. Although temporary, the new structure which contains DNA scaffold associated with antimicrobial proteins and proteases presents a platform for additional signaling or interactions with blood or tissue components. Studies on NET cytotoxicity towards endothelial and epithelial cells pointed out histones, MPO, NE and cathepsin $G$ as the main NET components involved in tissue destruction, whereas lungs are the main target as neutrophils reside in the lung longer than in other organs (Kolaczkowska \& Kubes, 2013).

\section{Lung diseases}

One consequence of chronic lung inflammation is the acute lung injury (ALI) and acute respiratory distress syndrome (ARDS) (Ware \& Matthay, 2000; Cheng \& Palaniyar, 2013). Additional risk factors (pathogenic infection, sepsis, chronic allergy as well as mechanical ventilation with high pressure, or transfusions) can lead to the injury of alveolar epithelial cells and increase the permeability of alveolar and capillary vessels (Ware \& Matthay, 2000). The activation and massive migration of neutrophils into the alveolar space, controlled by chemokines produced by epithelial cells, macrophages as well as neutrophils (Kasama et al., 2005), was observed in infectionrelated ALI/ARDS and in the sterile injury. A high concentration of stimulating factors present in the alveolar space promotes neutrophil activation and NET release (Fig. 2). On the other hand, the lower level of surfactant proteins (SP-A and SP-B) in the pulmonary surfactant layer, frequently observed in several inflammatory lung diseases, is responsible for a defective NETnucleic acid clearance (Douda et al., 2011; Nayak et al., 2012). Also, the proteinous NET components are potent lung injury factors. NE cleaves endothelial actin cytoskeleton, E-cadherin and VE-cadherin, increasing the permeability of the alveolar-capillary barrier. Moreover, $\mathrm{NE}$ induces apoptosis of epithelial cells and the release of proinflammatory cytokines (Saffarzadeh et al., 2012). Other proteinases (PR3, cathepsin $G$ ) are able to regulate 


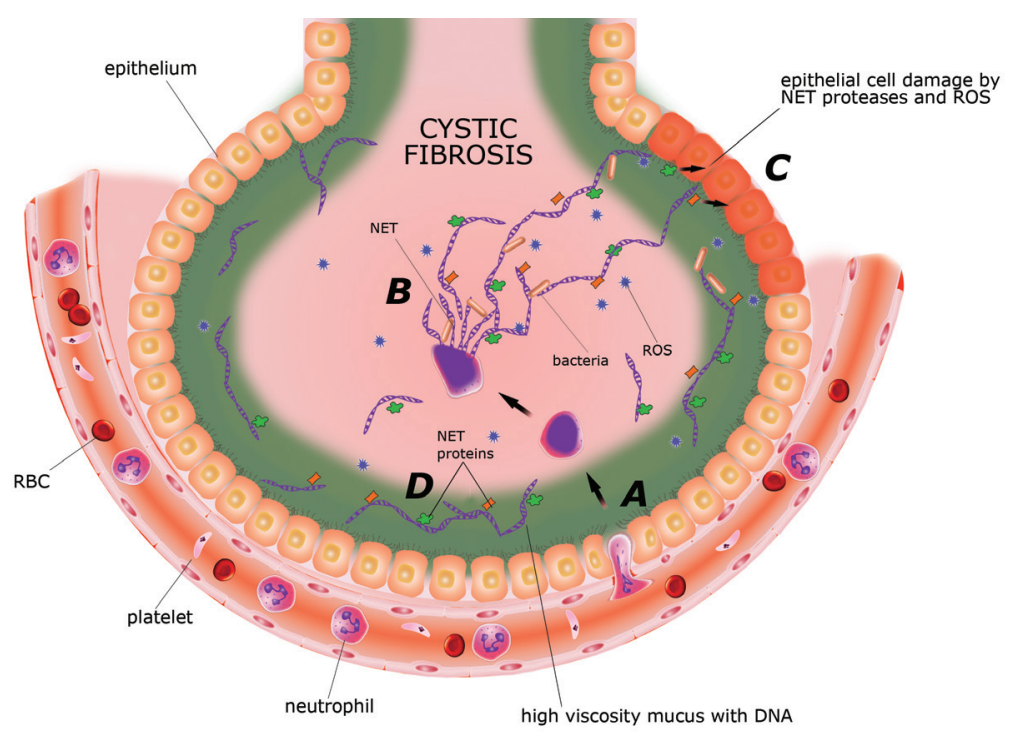

Figure 3. Role of NETs in increasing mucus viscosity.

In response to microbial infections neutrophils are recruited from the capillaries to alveoli $(\mathbf{A})$. CF patients produce a lot of mucus that is an excellent environment for bacteria development and can lead to chronic infection. Pathogens stimulate neutrophils to release NETs (B), an ineffective NET clearance causes epithelium injury by NET components (C) and increases mucus viscosity owing to the presence of DNA fibres (D).

the inflammatory process by activating proinflammatory and degrading anti-inflammatory proteins (Grommes \& Soehnlein, 2011). The antimicrobial peptide LL-37, detected in NET structures (Urban et al., 2009), presents cytotoxic and proapoptotic properties towards endothelial and epithelial cells (Aarbiou et al., 2006). In addition, ROS produced by MPO cause epithelial cell injury, which leads to apoptosis or necrosis (Grommes \& Soehnlein, 2011), besides ROS-promoted netosis (Nishinaka et al., 2011).

Similarly, the antibacterial and proinflammatory role of NETs was observed in cystic fibrosis (CF) patients (Fig. 3) $\mathrm{CF}$ is a lung disease resulting from a mutation in the cystic fibrosis transmembrane conductance regulator (Ratjen \& Grasemann, 2012). This mutation disrupts the normal transport of $\mathrm{Na}^{+}$and $\mathrm{Cl}^{-}$ions across epithelial cells causing dehydration and an increase in mucus viscosity, therefore hindering the clearance of mucus from the airways (Kaynar \& Shapiro, 2010; Ratjen \& Grasemann, 2012). Another factor responsible for high mucus viscosity is the presence of DNA in CF patient sputum (Henke \& Ratjen, 2007) that correlates with a high concentration of neutrophils and NET accumulation in CF lungs (Marcos et al., 2010). Increased levels of neutrophils lead to chronic neutrophilic inflammation, observed in CF patients, mostly caused by chronic bacterial and viral infections enhanced by conditions favoring microbial growth. Moreover, the NET production, additionally promoted by bacterial infection in CF airways, is often ineffective in bacterial killing as was presented for Pseudomonas aeruginosa (Marcos et al., 2010), and may facilitate bacterial airway colonisation and biofilm formation. Those conditions, often accompanied by a decreased SP-D level, lead to the increase in mucus viscosity and consequently to chronic inflammation (Cheng \& Palaniyar, 2013).

One of the currently proposed therapies for sputum viscosity reduction of which NET structures are the targets, is mucus DNA degradation by recombinant human DNase (rhDNase) (Henke \& Ratjen, 2007). However,
rhDNase is able to digest free chromatin more quickly than the DNA-protein complexes occurring in NETs. The role of $\mathrm{NE}$ in $\mathrm{CF}$ is ambiguous. On the one hand, Papayannopoulos et al. (2011) have presented data that NE enhances solubilization of sputum by degrading histones and facilitating the access for rhDNase. On the other hand, the liberated elastase as well as other proteolytic NET components can damage lung tissue and enhance the immune response by modulating the inflammatory factors. For example, active proteases are able to degrade SP-D, during the course of pathogenic infection (Cheng \& Palaniyar, 2013). However, NE in CF sputum is predominantly bound to DNA, which down-regulates its proteolytic activity but also precludes the inhibition by exogenous protease inhibitors (Dubois et al., 2012). Taking into account the benefits and problems resulting from the mucolytic therapy, it seems reasonable to introduce a combination therapy with rhDNase and protease inhibitors, which should offer the best compromise between lung tissue injury and easy mucus removal.

\section{Autoimmune diseases}

Autoimmune diseases such as small vessel vasculitis (SVV) or systemic lupus erythematosus (SLE) are other examples of the dark side of NET production and their ineffective clearance (Sangaletti et al., 2012; Brinkmann \& Zychlinsky, 2012; Darrah \& Andrade, 2012). Although the development of autoimmune disease is a highly complex process, the extracellular exposure of intracellular antigens is generally accepted as the fundamental step in the autoimmune response and the production of autoantibodies.

SVV is a systemic autoimmune disease which causes chronic inflammation of small blood vessels, e.g. in lungs, skin or kidneys, and is associated with the presence of anti-neutrophil cytoplasmic antibodies (ANCA). The main targets for ANCA are MPO (associated with microscopic polyangiitis) and PR3 (associated with Wegener's granulomatosis) (Kallenberg et al., 2006; Kessenbrock et al., 2009). Moreover, it was observed that the binding of ANCA to MPO or PR3 activated the neutrophils and promoted netosis (Kessenbrock et al., 2009). Established ANCA-MPO-complexes were also able to activate mDC. Treatment with DNase not only caused inhibition of NET formation but also prevented vessel inflammation, confirming the involvement of NETs in small vessel damage and SVV disease development (Sangaletti et al., 2012).

Neutrophils of SLE patients are activated and more likely to form NETs (Brinkmann \& Zychlinsky, 2012). This finding correlates with an increased level of circulating DNA in the plasma of SLE patients as well as with the presence of antibodies against NET-associated 
proteins (Tsokos, 2011). More than 70\% of NET components are potent autoantigens in SLE and other autoimmune diseases (Knight et al., 2012; Darrah \& Andrade, 2012).

The NET formation entails a unique histone modification in which arginine is converted to citrulline. The citrullinated histones seem not only to damage endothelial cells (Gupta et al., 2010) but can also constitute new autoantigens for the immune system (Darrah et al., 2012; Liu et al., 2012), which is additionally activated by prolonged exposure of NET proteins owing to the protection of DNA from degradation by DNase inhibitors (Lande et al., 2011). Such inhibitory effects can result from the protective function of the antibacterial peptide LL-37, another component of NETs (Lande et al., 2011), a high level of anti-NET antibodies or an increased deposition of the complement protein C1q (Leffler et al., 2012).

The breakdown of tolerance to the immune complexes (IC) formed in SLE causes multi-organ inflammation and damage (Garcia-Romo et al., 2011). Moreover, IC stimulate other neutrophils to produce NET (Knight et al., 2012) and activate plasmacytoid dendritic cells through Toll-like receptors (TLR7) to secrete interferon (IFN- $\alpha$ ) (Crispín et al., 2010; Tsokos, 2011; Garcia-Romo et al., 2011). All the proposed models of the interplay between netosis and autoimmune diseases are discussed in detail by (Darrah \& Andrade, 2012).

\section{Thrombosis}

Deep vein thrombosis (DVT) is a disease that may be complicated by pulmonary or venous embolism and leads to serious multiple organ ischemia. The factors that contribute to thrombosis, called Virchow's triad, include: stasis (low vascular blood flow), endothelial or vessel wall damage and hypercoagulability. Stasis leads to platelet deposition, an increase in the concentration of procoagulant factors and thrombus formation (Line, 2001). Ad- ditionally, endothelium may be activated by local hypoxia (Hamer et al., 1981). Activated endothelium releases von Willebrand factor (vWF) which is necessary for platelet recruitment and adhesion (Brill et al., 2011). Moreover, activated endothelium produces compounds that, upon contact with neutrophils, stimulate netosis which, in turn, promotes endothelial damage (Fig. 4) (Gupta et al., 2010). Application of deoxyribonuclease 1 (DNase 1) in a murine model of DVT protected mice from DVT and revealed that the cleavage of NETs by DNase1 prevents the cascade of events leading to thrombosis (Brill et al., 2012).

Thrombosis can also be initiated by the release of the tissue factor (TF) as well as by cytokines produced during inflammatory processes associated with infections, autoimmune disorders and cancer (Line, 2001; Fuchs et al., 2012). TF binds factor VII to give TF-FVIIa complex which is able to activate the coagulation cascade with clot formation (Wolberg et al., 2012). A recent finding that TF can be produced by neutrophils and expulsed to the vein during NET formation is the first evidence that neutrophils and netosis provide an interface between inflammation and thrombosis (Von Brühl et al., 2012; Fuchs et al., 2012). In the model studies, the neutrophils, after stimulation with P-selectin or fMLP (but not PMA), expressed TF intracellularly and only a small fraction of TF translocated to the cell surface. The same effects were observed for neutrophils isolated from patients with ARDS (Kambas et al., 2008). However, neutrophils from patients with sepsis contain a large amount of TF delivered and released by NETs (Kambas et al., 2012). Additionally, NE present in NETs can regulate coagulation pathway by proteolytic cleavage of TF pathway inhibitors and enhancement of Factor Xa activity (Steppich et al., 2008).

NETs, assisted by DNA and histones, also bind factor XII thereby stimulating fibrin formation via the intrinsic coagulation pathway (Von Brühl et al., 2012).

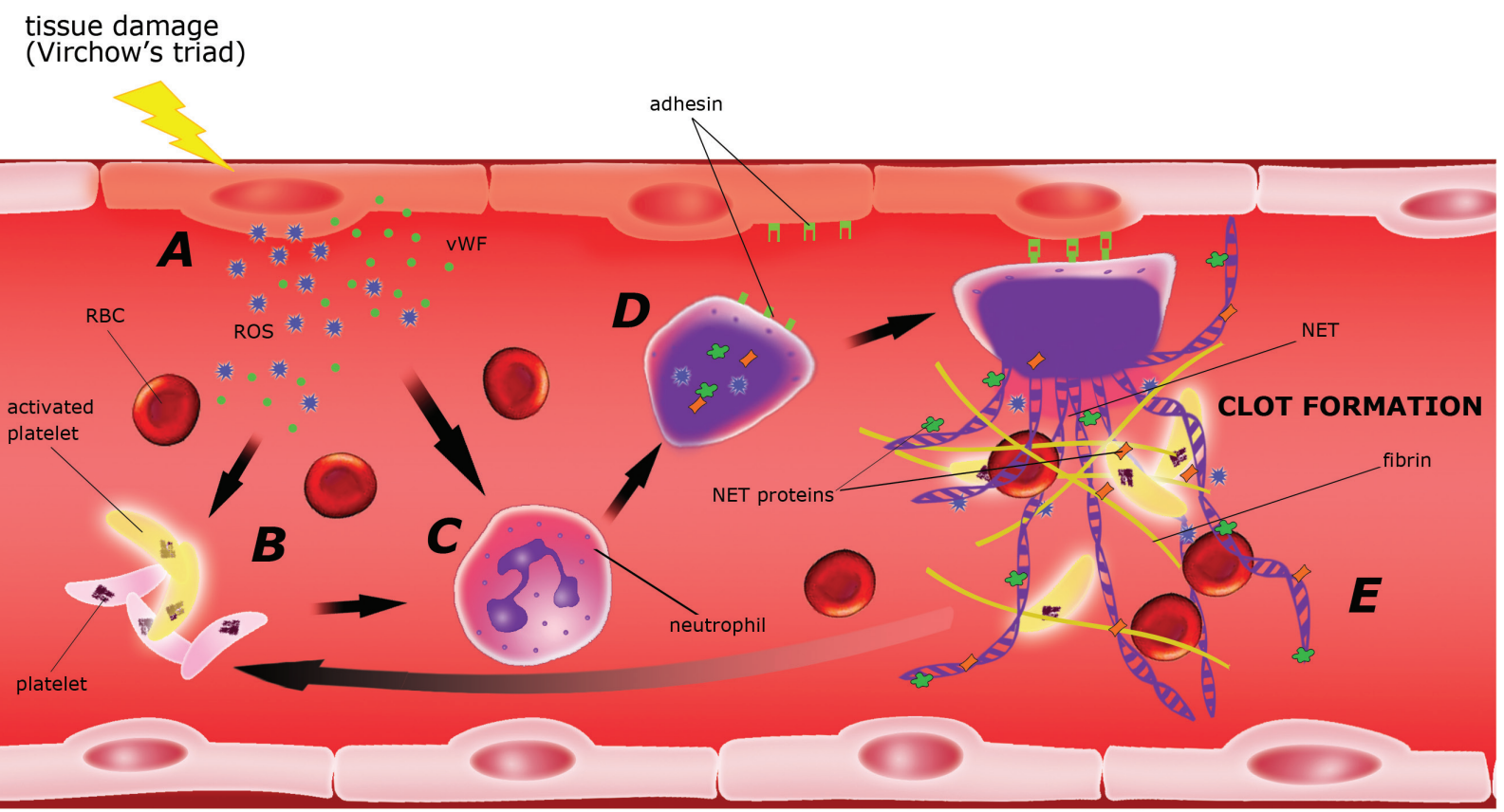

Figure 4. Clot formation after blood vessel injury.

The damage of blood vessels causes the release of von Willebrand factor (vWF) by endothelial cells (A), which in turn activate platelets (B) and neutrophils. Moreover, neutrophils can be stimulated by activated platelets (C). This leads to changes in nucleus (D) and in consequence to NET release. NETs bind the blood cells (e.g. RBC, platelets) and proteins, such as fibrin, leading to clot formation (E). 
Another way of NET contribution to thrombus formation is platelet entrapment and activation (Ma \& Kubes, 2008). NET fibres bind platelets directly or indirectly, and support their aggregation (Fuchs et al., 2011). The first step of platelet binding involves Toll- like receptors (Semeraro et al., 2011) or is based on electrostatic interaction between histones located in NETs and phospholipids or carbohydrates of platelets. In another model, the platelet-NET interactions were proposed to be mediated by adhesion molecules: vWF, fibronectin or fibrinogen (Fuchs et al., 2010; Brill et al., 2012). Additionally, platelets bound to NETs can be activated by components of traps, especially by histones, through stimulation of the calcium flux (Fuchs et al., 2011) or by neutrophil proteases, which proteolytically activate the platelet receptors (Si-Tahar et al., 1997). This process may be accelerated, as activated platelets cause further NET release that increases endothelial permeability (Fuchs et al., 2010; Brill et al., 2012).

Based on the observations described above we can conclude that neutrophils, platelets and endothelial cells effectively interact with the coagulation factors. The synergistic effect of different NET functions, i.e., antimicrobial and prothrombotic, seems to be essential to preserve homeostasis in infectious disease, especially in sepsis.

\section{NETs and cancer}

A hypothesis that netosis has some significance in cancer has arisen from recent studies showing that neutrophils, found in large quantities in plasma of cancer patients, possessed pro- and anti-tumour activities (Souto et al., 2011). On the one hand, neutrophils favour migration of cancer cells by directly interacting with them (Huh et al., 2010), promote tumour growth by secreting matrix metalloproteinases (Acuff et al., 2006) or tumour angiogenesis and neovascularization (Masson et al., 2005) as well as establish the environment for metastatic cancer cells (Kowanetz et al., 2010). On the other hand, activated neutrophils exert cytotoxic effects on tumour cells by releasing ROS or defensins (Granot et al., 2011). Recently, it has been observed that the granulocyte colony-stimulating factor (G-CSF), produced by many tumours and found in the circulation of cancer patients, not only influenced the mobilisation and activation of neutrophils but also triggered NET formation (Demers et al., 2012). Moreover, in mice at the late-stage of cancer a high quantity of plasma DNA and citrullinated histones was detected. Their production correlated with an increased level of neutrophil markers and micro-thrombi formation in the lung (Demers et al., 2012). This is the first evidence of correlations between cancer or metastasis and NET formation and thrombosis. However, at the actual stage of our knowledge, it is difficult to decide whether netosis plays a pro- or anti-tumorigenic role. It was speculated that NET components like MPO, proteinases and histones could be cytotoxic to tumour cells and inhibit their growth.

Moreover, it was also suggested that NETs could serve as a scaffold for the capture of tumour cells and, thereby, prevent their further dissemination (BergerAchituv et al., 2013). Alternatively, NETs, through the action of their proteinous components could promote extravasation and metastasis. Finally, NETs could adhere to the metastatic cells and by recruiting platelets could protect them and attenuate the immune response (Demers \& Wagner, 2013).

Nevertheless, better understanding of the function of NETs in tumour progression can lead in the future to the development of new prognostic markers or anti-cancer therapies.

\section{CONCLUSION}

NET production by neutrophils plays an essential role in immune response to infection. The chromatin scaffold binds pathogens preventing their dissemination and limiting the inflammation area while the components of NETs very efficiently kill the trapped pathogens by oxidative and non-oxidative mechanisms. Regardless of antimicrobial function, NETs participate also in many non-infectious diseases, autoimmune and inflammatory disorders, including chronic lung disease, sepsis, and vascular disorders. The increased autoreactivity towards NET constituents is a result of excessive netosis or diminished NET clearance. Although a range of biological events activating NET release is currently under excessive exploration, the mechanisms of its regulation are still unknown. The discovery of the association of netosis with pathophysiological and immunological processes has not only helped to understand NET functioning during specific disease states or microbial infections but also may potentially lead to the discovery of new effective therapeutic agents.

\section{Acknowledgements}

This work was supported by the grant no 2012/05/B/ NZ1/00003 from the National Science Centre (Poland).

We thank Professor Andrzej Kozik for critical discussion during the manuscript preparation.

\section{REFERENCES}

Aarbiou J, Tjabringa GS, Verhoosel RM, Ninaber DK, White SR, Peltenburg LTC, Rabe KF, Hiemstra PS (2006) Mechanisms of cell death induced by the neutrophil antimicrobial peptides alphadefensins and LL-37. Inflamm Res 55: 119-127.

Acuff HB, Carter KJ, Fingleton B, Gorden DL, Matrisian LM (2006) Matrix metalloproteinase- 9 from bone marrow-derived cells contributes to survival but not growth of tumor cells in the lung microenvironment. Cancer Res 66: 259-266.

Beiter K, Wartha F, Albiger B, Normark S, Zychlinsky A,HenriquesNormark B (2006) An endonuclease allows Streptococcus pneumoniae to escape from neutrophil extracellular traps. Curr Biol 16: 401-407.

Berger-Achituv S, Brinkmann V, Abed UA, Kühn LI, Ben-Ezra J, Elhasid R, Zychlinsky A (2013) A proposed role for neutrophil extracellular traps in cancer immunoediting. Frontiers Immunol 4: 48.

Brill A, Fuchs T, a Chauhan AK, Yang JJ, De Meyer SF, Köllnberger M, Wakefield T, Lämmle B, Massberg S, Wagner DD (2011) von Willebrand factor-mediated platelet adhesion is critical for deep vein thrombosis in mouse models. Blood 117: 1400-1407.

Brill A, Fuchs TA, Savchenko AS, Thomas GM, Martinod K, De Meyer SF, Bhandari AA, Wagner DD (2012) Neutrophil extracellular traps promote deep vein thrombosis in mice. J Thrombosis Haemostasis 10: 136-144.

Brinkmann V, Reichard U, Goosmann C, Fauler B, Uhlemann Y, Weiss DS, Weinrauch Y, Zychlinsky A (2004) Neutrophil extracellular traps kill bacteria. Science 303: 1532-1535.

Brinkmann V, Zychlinsky A (2012) Neutrophil extracellular traps: is immunity the second function of chromatin? I Cell Biol 198: 773-783.

Von Brühl M-L, Stark K, Steinhart A, Chandraratne S, Konrad I, Lorenz M, Khandoga A, Tirniceriu A, Coletti R, Köllnberger M et al. (2012) Monocytes neutrophils and platelets cooperate to initiate and propagate venous thrombosis in mice in vivo. I Exp Med 209: 819-835.

Cheng OZ, Palaniyar N (2013) NET balancing: a problem in inflammatory lung diseases. Frontiers Immunol 4: 1.

Cho JH, Sung BH, Kim SC (2009) Buforins: histone H2A-derived antimicrobial peptides from toad stomach. Biochim Biophys Acta 1788: 1564-1569.

Crispín JC, Liossis SC, Kis-Toth K, Lieberman LA, Kyttaris VC, Juang Y, Tsokos GC (2010) Pathogenesis of human systemic lupus erythematosus: recent advances. Trends Mol Med 16: 47-57. 
Darrah E, Andrade F (2012) NETs: the missing link between cell death and systemic autoimmune diseases? Frontiers Immunol 3: 428.

Darrah E, Rosen A, Giles JT, Andrade F (2012) Peptidylarginine deiminase 2,3 , and 4 have distinct specificities against cellular substrates: novel insights into autoantigen selection in rheumatoid arthritis. Ann Rheumatic Dis 71: 92-98.

Demers M, Krause DS, Schatzberg D, Martinod K, Voorhees JR, Fuchs TA, Scadden DT, Wagner DD (2012) Cancers predispose neutrophils to release extracellular DNA traps that contribute to cancer-associated thrombosis. Proc Natl Acad Sci USA 109: 1307613081.

Demers M, Wagner DD (2013) Neutrophil extracellular traps: A new link to cancer-associated thrombosis and potential implications for tumor progression. Oncoimmunol 2: e22946.

Douda DN, Jackson R, Grasemann H, Palaniyar N (2011) Innate immune collectin surfactant protein D simultaneously binds both neutrophil extracellular traps and carbohydrate ligands and promotes bacterial trapping. I Immunol 187: 1856-1865.

Dubois AV, Gauthier A, Bréa D, Varaigne F, Diot P, Gauthier F, Attucci S (2012) Influence of DNA on the activities and inhibition of neutrophil serine proteases in cystic fibrosis sputum. Am J Respir Cell Mol Biol 47: 80-86.

Farnaud S, Evans RW (2003) Lactoferrin - a multifunctional protein with antimicrobial properties. Mol Immunol 40: 395-405.

Fuchs T, a Abed U, Goosmann C, Hurwitz R, Schulze I, Wahn V, Weinrauch Y, Brinkmann V, Zychlinsky A (2007) Novel cell death program leads to neutrophil extracellular traps. J Cell Biol 176: 231 241 .

Fuchs T, a Bhandari A, Wagner DD (2011) Histones induce rapid and profound thrombocytopenia in mice. Blood 118: 3708-3714.

Fuchs TA, Brill A, Duerschmied D, Schatzberg D, Monestier M, Myers DD, Wrobleski SK, Wakefield TW, Hartwig JH, Wagner DD (2010) Extracellular DNA traps promote thrombosis. Proc Natl Acad Sc USA 107: 15880-15885.

Fuchs TA, Brill A, Wagner DD (2012) Neutrophil extracellular trap (NET) impact on deep vein thrombosis. Arteriosclerosis Thrombosis Vascular Biol 32: 1777-1783.

Garcia-Romo GS, Caielli S, Vega B, Connolly J, Allantaz F, Xu Z, Punaro M, Baisch J, Guiducci C, Coffman RL et al. (2011) Netting neutrophils are major inducers of type I IFN production in pediatric systemic lupus erythematosus. Sci Translat Med 3: 73ra20.

Goldmann O, Medina E (2013) The expanding world of extracellular traps: not only neutrophils but much more. Frontiers Immunol 3: 420.

Granot Z, Henke E, Comen EA, King TA, Norton L, Benezra R (2011) Tumor entrained neutrophils inhibit seeding in the premetastatic lung. Cancer Cell 20: 300-314.

Grommes J, Soehnlein O (2011) Contribution of neutrophils to acute lung injury. Mol Med 17: 293-307.

Guimarães-Costa AB, Nascimento MTC, Wardini AB, Pinto-da-Silva LH, Saraiva EM (2012) ETosis: A Microbicidal Mechanism beyond Cell Death. J Parasitol Res 2012: 929743.

Gupta AK, Joshi MB, Philippova M, Erne P, Hasler P, Hahn S, Resink TJ (2010) Activated endothelial cells induce neutrophil extracellular traps and are susceptible to NETosis-mediated cell death. FEBS Lett 584: 3193-3197.

Hamer JD, Malone PC, Silver IA (1981) The PO2 in venous valve pockets: its possible bearing on thrombogenesis. British J Surgery 68 : 166-170.

Henke MO, Ratjen F (2007) Mucolytics in cystic fibrosis. Paediatric Respiratory Rev 8: 24-29.

Huh SJ, Liang S, Sharma A, Dong C, Robertson GP (2010) Transiently entrapped circulating tumor cells interact with neutrophils to facilitate lung metastasis development. Cancer Res 70: 6071-6082.

Kallenberg CGM, Heeringa P, Stegeman C (2006) Mechanisms of Disease: pathogenesis and treatment of ANCA-associated vasculitides. Nature Clin Practice. Rheumatology 2: 661-670.

Kambas K, Markiewski MM, Pneumatikos IA, Rafail SS, Theodorou V, Konstantonis D, Kourtzelis I, Doumas MN, Magotti P, Deangelis RA et al. (2008) C5a and TNF- alpha up-regulate the expression of tissue factor in intra-alveolar neutrophils of patients with the acute respiratory distress syndrome. J Immunol 180: 7368-7375.

Kambas K, Mitroulis I, Ritis K (2012) The emerging role of neutrophils in thrombosis- the journey of TF through NETs. Frontiers Immunol 3: 385.

Kasama T, Miwa Y, Isozaki T, Odai T, Adachi M, Kunkel SL (2005) Neutrophil-derived cytokines: potential therapeutic targets in inflammation. Current Drug Targets. Inflammation Allergy 4: 273-279.

Kaynar M, Shapiro SD (2010) NET loss of air in cystic fibrosis. Nature Med 16: 967-969.

Kessenbrock K, Krumbholz M, Schönermarck U, Back W, Gross WL, Werb Z, Gröne H, Brinkmann V, Jenne DE (2009) Netting neutrophils in autoimmune small-vessel vasculitis. Nature Med 15: 623-625.

Knight JS, Carmona-Rivera C, Kaplan MJ (2012) Proteins derived from neutrophil extracellular traps may serve as self-antigens and mediate organ damage in autoimmune diseases. Frontiers Immunol 3: 380.
Kolaczkowska E, Kubes P (2013) Neutrophil recruitment and function in health and inflammation. Nature Rev. Immunol 13: 159-175.

Kowanetz M, Wu X, Lee J, Tan M, Hagenbeek T, Qu X, Yu L, Ross J, Korsisaari N, Cao T et al. (2010) Granulocyte-colony stimulating factor promotes lung metastasis through mobilization of Ly6G + Ly6C+ granulocytes. Proc Natl Acad Sci USA 107: 21248-21255.

Lande R, Ganguly D, Facchinetti V, Frasca L, Conrad C, Gregorio J, Meller S, Chamilos G, Sebasigari R, Riccieri V et al. (2011) Neutrophils activate plasmacytoid dendritic cells by releasing self-DNApeptide complexes in systemic lupus erythematosus. Sci Translational Med 3: 73ra19.

Leffler J, Martin M, Gullstrand B, Tydén H, Lood C, Truedsson L, Bengtsson AA, Blom AM (2012) Neutrophil extracellular traps that are not degraded in systemic lupus erythematosus activate complement exacerbating the disease. I Immunol 188: 3522-3531.

Leshner M, Wang S, Lewis C, Zheng H, Chen X. A, Santy L,Wang Y (2012) PAD4 mediated histone hypercitrullination induces heterochromatin decondensation and chromatin unfolding to form neutrophil extracellular trap-like structures. Frontiers Immunol 3: 307.

Li P, Li M, Lindberg M. R, Kennett M. J, Xiong N, Wang Y (2010) PAD4 is essential for antibacterial innate immunity mediated by neutrophil extracellular traps. I Exp Med 207: 1853-1862.

Line BR (2001) Pathophysiology and diagnosis of deep venous thrombosis. Seminars Nuclear Med 31: 90-101.

Liu CL, Tangsombatvisit S, Rosenberg JM, Mandelbaum G, Gillespie EC, Gozani OP, Alizadeh A, Utz PJ (2012) Specific post-translational histone modifications of neutrophil extracellular traps as immunogens and potential targets of lupus autoantibodies. Arthritis Res Therapy 14: R25.

Lu T, Kobayashi SD, Quinn MT, Deleo FR (2012) A NET Outcome. Frontiers Immunol 3: 365.

Ma A, C, Kubes P (2008) Platelets neutrophils and neutrophil extracellular traps (NETs) in sepsis. I Thrombosis Haemostasis 6: 415-420.

Marcos V, Zhou Z, Yildirim AO, Bohla A, Hector A, Vitkov L, Wiedenbauer E-M, Krautgartner WD, Stoiber W, Belohradsky BH et al. (2010) CXCR2 mediates NADPH oxidase-independent neutrophil extracellular trap formation in cystic fibrosis airway inflammation. Nature Med 16: 1018-1023.

Masson V, De la Ballina LR, Munaut C, Wielockx B, Jost M, Maillard C, Blacher S, Bajou K, Itoh T, Itohara S et al. (2005) Contribution of host MMP-2 and MMP-9 to promote tumor vascularization and invasion of malignant keratinocytes. FASEB J 19: 234-236.

Méndez-Samperio P (2010) The human cathelicidin hCAP18/LL-37: a multifunctional peptide involved in mycobacterial infections. Peptides 31: $1791-1798$.

Metzler KD, Fuchs T, a Nauseef WM, Reumaux D, Roesler J, Schulze I, Wahn V, Papayannopoulos V, Zychlinsky A (2011) Myeloperoxidase is required for neutrophil extracellular trap formation: implications for innate immunity. Blood 117: 953-959.

Nayak A, Dodagatta-Marri E, Tsolaki AG, Kishore U (2012) An Insight into the Diverse Roles of Surfactant Proteins SP-A and SP-D in Innate and Adaptive Immunity. Frontiers Immunol 3: 131.

Neeli I, Khan SN, Radic M (2008) Histone deimination as a response to inflammatory stimuli in neutrophils. J Immunol 180: 1895-1902.

Neeli I, Radic M (2013) Opposition between PKC isoforms regulates histone deimination and neutrophil extracellular chromatin release. Frontiers Immunol 4: 38.

Nishinaka Y, Arai T, Adachi S, Takaori-Kondo A, Yamashita K (2011) Singlet oxygen is essential for neutrophil extracellular trap formation. Biochem Biophys Res Commun 413: 75-79.

Papayannopoulos V, Metzler KD, Hakkim A, Zychlinsky A (2010) Neutrophil elastase and myeloperoxidase regulate the formation of neutrophil extracellular traps. I Cell Biol 191: 677-691.

Papayannopoulos V, Staab D, Zychlinsky A (2011) Neutrophil elastase enhances sputum solubilization in cystic fibrosis patients receiving DNase therapy. PloS One 6: e28526.

Papayannopoulos V, Zychlinsky A (2009) NETs: a new strategy for using old weapons. Trends Immunol 30: 513-521.

Parker H, Winterbourn CC (2012) Reactive oxidants and myeloperoxidase and their involvement in neutrophil extracellular traps. Frontiers Immunol 3: 424.

Pilsczek FH, Salina D, Poon KKH, Fahey C, Yipp BG, Sibley CD, Robbins SM, Green FHY, Surette MG, Sugai M et al. (2010) A novel mechanism of rapid nuclear neutrophil extracellular trap formation in response to Staphylococcus aureus. J Immunol 185: 7413-7425.

Ratjen F, Grasemann H (2012) New therapies in cystic fibrosis. Curr Pharmaceutical Desion 18: 614-627.

Saffarzadeh M, Juenemann C, Queisser M, Lochnit G, Barreto G, Galuska SP, Lohmeyer J, Preissner KT (2012) Neutrophil extracellular traps directly induce epithelial and endothelial cell death: a predominant role of histones. PloS One 7: e32366.

Sangaletti S, Tripodo C, Chiodoni C, Guarnotta C, Cappetti B, Casalini P, Piconese S, Parenza M, Guiducci C, Vitali C et al. (2012) Neutrophil extracellular traps mediate transfer of cytoplasmic neutrophil antigens to myeloid dendritic cells toward ANCA induction and associated autoimmunity. Blood 120: 3007-3018. 
Semeraro F, Ammollo CT, Morrissey JH, Dale GL, Friese P, Esmon NL, Esmon CT (2011) Extracellular histones promote thrombin generation through platelet- dependent mechanisms: involvement of platelet TLR2 and TLR4. Blood 118: 1952-1961.

Si-Tahar M, Pidard D, Balloy V, Moniatte M, Kieffer N, Van Dorsselaer A, Chignard M (1997) Human neutrophil elastase proteolytically activates the platelet integrin alphaIIbbeta3 through cleavage of the carboxyl terminus of the alphalIb subunit heavy chain. Involvement in the potentiation of platelet aggregation. J Biol Chem 272: 11636-11647.

Souto JC, Vila L, Brú A (2011) Polymorphonuclear neutrophils and cancer: intense and sustained neutrophilia as a treatment against solid tumors. Med Res Rev 31: 311-363.

Steppich B, Seitz I, Busch G, Stein A, Ott I (2008) Modulation of tissue factor and tissue factor pathway inhibitor-1 by neutrophil proteases. Thrombosis Haemostasis 100: 1068-1075.

Tsokos GC (2011) Systemic lupus erythematosus. New England J Med 365: 2110-2121.

Urban CF, Ermert D, Schmid M, Abu-Abed U, Goosmann C, Nacken W, Brinkmann V, Jungblut PR, Zychlinsky A (2009) Neutrophil extracellular traps contain calprotectin a cytosolic protein complex involved in host defence against Candida albicans. PLoS Pathogens 5 e1000639.

Wang Y, Li M, Stadler S, Correll S, Li P, Wang D, Hayama R, Leonelli L, Han H, Grigoryev S et al. (2009) Histone hypercitrullination mediates chromatin decondensation and neutrophil extracellular trap formation. J Cell Biol 184: 205-213.

Ware LB, Matthay MA (2000) The acute respiratory distress syndrome. New England J Med 342: 1334-1349.

Wartha F, Beiter K, Normark S, Henriques-Normark B (2007) Neutrophil extracellular traps: casting the NET over pathogenesis. Curr Opin Microbiol 10: 52-56.

Wolberg AS, Aleman MM, Leiderman K, Machlus KR (2012) Procoagulant activity in hemostasis and thrombosis: Virchow's triad revisited. Anesthesia Analgesia 114: 275-285.

Yipp BG, Petri B, Salina D, Jenne CN, Scott BNV, Zbytnuik LD, Pittman K, Asaduzzaman M, Wu K, Meijndert HC et al. (2012) Infection-induced NETosis is a dynamic process involving neutrophil multitasking in vivo. Nat Med 18: 1386-1393. 